\title{
Thyroid Research in India: A Scientometric Assessment of Publications Output during 2007-16
}

\author{
B.M.Gupta', K K Mueen Ahmed ${ }^{2}$ and Ritu Gupta ${ }^{3}$
}

B.M.Gupta ${ }^{1}$, K K Mueen Ahmed ${ }^{2}$ and Ritu Gup$\mathrm{ta}^{3}$

'1173 Sector 15, Panchkula 134 113, Haryana, INDIA. ${ }^{2}$ Phcog.Net and SciBiolMed.Org, Bengaluru, Karnataka, INDIA.

${ }^{3} 1 \mathrm{~K} / \mathrm{A}$ Arjun Nagar, Safdarjang Enclave, New Delhi 110029, INDIA.

\section{Correspondence}

Ritu Gupta

1K/A Arjun Nagar, Safdarjang Enclave, New Delhi 110029, INDIA.

Mobile no: 9711563995

Email: ritu7648@gmail.com

\section{History}

- Submission Date: 14-04-2017

- Revised Date: 23-05-2017

- Accepted Date: 23-05-2017

\section{DOI : 10.5530/ijmedph.2017.3.28}

Article Available online

http://www.ijmedph.org/v7/i3

\section{Copyright}

(C) 2017 Phcog.Net. This is an openaccess article distributed under the terms of the Creative Commons Attribution 4.0 International license.

\begin{abstract}
The paper examines 2483 global publications on thyroid research in India, as covered in Scopus database during 2007-16, experiencing an annual average growth rate of $10.76 \%$ and qualitative citation impact averaged to 4.75 citations per paper. India's share of internationally collaborative papers (ICP) was $8.82 \%$ during 2007-16, which increased from $8.19 \%$ to $9.21 \%$ from $2007-11$ to $2012-16$. The top 10 most productive countries individually contributed global share from $3.67 \%$ to $25.88 \%$, with largest global publication share coming from USA $(25.88 \%)$, followed by Italy $(8.15 \%)$, etc. Together, the 10 most productive countries accounted for $73.75 \%$ share of global publication output during 2007-16. Medicine accounts for the largest share $(77.57 \%)$ in Indian thyroid research, followed by biochemistry, genetics \& molecular biology (23.60\%), pharmacology, toxicology \& pharmaceutics $(8.46 \%)$, agricultural \& biological sciences (3.34\%) and neurosciences (2.54\%) during 2007-16. The top 15 most productive research organizations and the authors collectively contributed $31.70 \%$ and $11.24 \%$ respectively as their global publication share and $34.72 \%$ and $17.58 \%$ respectively as their global citation share during 2007-16. The journals accounted $23.23 \%$ share of total journal publication output during 2007-16, increasing from $19.62 \%$ to $25.66 \%$ between $2007-11$ and 2012-16. Of the total thyroid research output from India, the top 13 highly cited publications registered citations from 83 to 422 and they together received 2023 citations, with 155.61 citations per paper. These 13 highly cited papers were published in 11 journals, of which 2 papers were published in American Journal of Transplantation and 1 paper each in 10 other journals.

Key words: Thyroid research, Indian publications, Scientometrics, Bibliometrics.
\end{abstract}

\section{INTRODUCTION}

Thyroid disorders are conditions that affect the thyroid gland, a butterfly-shaped gland in the front of the neck. The thyroid has important roles to regulate numerous metabolic processes throughout the body. Different types of thyroid disorders affect either its structure or function. The thyroid gland is located below the Adam's apple wrapped around the trachea (windpipe). A thin area of tissue in the gland's middle, known as the isthmus, joins the two thyroid lobes on each side. The thyroid uses iodine to produce vital hormones. Thyroxine, also known as T4, is the primary hormone produced by the gland. After delivery via the bloodstream to the body's tissues, a small portion of the T4 released from the gland is converted to triiodothyronine (T3), which is the most active hormone. The function of the thyroid gland is regulated by a feedback mechanism involving the brain. When thyroid hormone levels are low, the hypothalamus in the brain produces a hormone known as thyrotropin releasing hormone (TRH) that causes the pituitary gland (located at the base of the brain) to release thyroid stimulating hormone (TSH). TSH stimulates the thyroid gland to release more T4. Since the thyroid gland is controlled by the pituitary gland and hypo- thalamus, disorders of these tissues can also affect thyroid function and cause thyroid problems. ${ }^{1}$

Thyroid diseases are among the commonest endocrine disorders worldwide. In India it has been estimated that about 42 million people suffer from thyroid disorders, majorly because the entire population, and not just the Himalayan belt, is prone to iodine deficiency disorders (IDD) due to deficiency of iodine in the soil of the subcontinent. The overall prevalence of IDD is above $10 \%$ in India. IDD in pregnancy can lead to spontaneous abortions and stillbirths. It can have profound effects on the growing fetus and lead to congenital goiter and cretinism in the newborn. However, the effects can be observed during childhood when it can affect the growth and development of children, as also during adulthood when it can affect the productivity. Hypothyroidism and Autoimmune thyroiditis are common disorders in India, and over the years its incidences seem to be on the rise. ${ }^{2}$ Thyroid diseases are different from other diseases in terms of their ease of diagnosis, accessibility of medical treatment, and the relative visibility that even a small swelling of the thyroid offers to the
Cite this article : Gupta BM, Mueen Ahmed KK, Gupta R. Thyroid Research in India: A Scientometric Assessment of Publications Output during 2007-16. Int J Med Public Health. 2017;7(3):134-41. 
treating physician. Early diagnosis and treatment remains the cornerstone of management. ${ }^{3}$

\section{Literature Review}

Only one study is available by Bhutani, Verma and Kalra [4], which quantitatively analyze 210 publications (appearing in 23 main issues and 1 special issue) of the journal entitled "Thyroid Research \& Practice" during 2006-13. The articles covered in this journal are classified by type of articles, authorship pattern and by sub-specialties, origin of authors from India and abroad and contribution by academic and non-academic institutions.

\section{OBJECTIVES}

The main objectives of this study are to study the performance of Indian thyroid research during 2007-16, based on publications output covered in Scopus database. In particular, the study focuses on the following objectives: (i) To study the growth of world research output, contribution of top 10 most productive countries, Indian research output and its citation impact; (ii) To study the share of international collaboration papers in India's overall research output and contribution of important international collaborative partner countries; (iii) To study the Indian research output by broad subject areas, by sub-fields and the trends of research by identifying important keywords; (iv) To study the publication productivity and citation impact of top 15 most productive organizations and authors; (vi) To study the modes of communication in research and identification of core journals and (vii) to study the characteristics of top 13 highly cited papers.

\section{METHODOLOGY}

The study retrieved and downloaded 10-year publication data of the world output in thyroid research from the Scopus database (http://www. scopus.com) covering the period 2007-16. Keywords, such as "thyroid" was incorporated in the search string and qualified these keyword with "keyword tag", "Article Title tag" and "Source Title tag", and in addition incorporated in this search string the period '2007-16' within "date range tag". Finally this search string was applied for searching global publication data on thyroid research. The search string was subsequently refined by "subject area tag", "country tag", "source title tag", "journal title name" and "affiliation tag", to get data/information on the distribution of publications output by subject, collaborating countries, author-wise, organization-wise and journal-wise, etc. For citation data, citations to publications were also collected from date of publication till 6 February 2017.

(KEY(thyroid) OR TITLE(thyroid) OR SRCTITLE(thyroid)) AND PUBYEAR > 2006 AND PUBYEAR $<2017$

(KEY(thyroid) OR TITLE(thyroid)OR SRCTITLE(thyroid)) AND PUBYEAR > 2006 AND PUBYEAR < 2017 AND ( LIMIT-TO ( AFFILCOUNTRY,'India” ) )

\section{ANALYSIS}

The total global and Indian research output in field of thyroid research cumulated to 64992 and 2483 publications in 10 years during 2007-16. The annual output of the world and India in thyroid research research increased from 5489 and 140 in the year 2007 to 5560 and 323 publications in the year 2016, registering $0.67 \%$ and $10.96 \%$ growth per annum. The cumulative world and Indian output in thyroid research in 5 years 2007-11 increased from 30010 and 952 to 34982 and 1531 publications during succeeding 5-year period 2012-16, registering $16.17 \%$ and $60.82 \%$ quinquennial growth. India's share in global output on thyroid research was $8.82 \%$, which increased from $8.19 \%$ to $9.21 \%$ from $2007-11$ to 2012-16. Of the total global publications output in thyroid research, $76.72 \%$ (1905) was published as articles, 9.63\% (239) as letters, 8.26\% (205) as reviews, $1.77 \%$ (44) as conference papers, $1.25 \%(31)$ as notes, $1.055(26)$ as editorials and the rest as book chapters, articles in press and short surveys. The citation impact of Indian publications on thyroid research averaged to 4.75 citations per publication (CPP) during 200716; five-yearly impact averaged to $8.93 \mathrm{CPP}$ for the period 2007-11 which declined to 2.15 CPP in the succeeding five-year 2012-16 (Table 1).

\section{International Collaboration}

India's share of internationally collaborative papers (ICP) was $8.82 \%$ during 2007-16, which increased from $8.19 \%$ to $9.21 \%$ from $2007-11$ to 2012-16. Among the leading countries contributing to internationally collaborative papers, USA topped the list with $49.32 \%$ share, followed by Italy and U.K. (10.50\% share each), Germany (7.76\% share), France, Japan and Netherlands (6.85\% share each), Australia (6.39\% share), China (5.94\% share) and Saudi Arabia (5.02\% share) during 2007-16. The international collaborative publications share increased by $14.87 \%$ by USA,3.82\% by Saudi Arabia, $32.37 \%$ by Italy, $2.10 \%$ by Germany and $0.68 \%$ by Netherlands, as against decrease by $7.58 \%$ in U.K., $6.00 \%$ by Australia, $5.29 \%$ by France, $2.73 \%$ by China and $1.31 \%$ by Japan from 2007-11 to 2012-16 (Table 1-2).

\section{Top 10 Most Productive Countries in Thyroid Research}

The global research output in the field of thyroid research had originated from as many as 152 countries in the world during 2007-16. Top 10 most productive countries in thyroid research had contributed 2383 to 16823 publications each during 2007-16 (Table 3). Of the 152 countries, 102 contributed 1-10 papers each, 47 countries 11-50 papers each,28 countries 51-100 papers each and 1 country 159 papers each. Top 10 most productive countries in thyroid research accounted for $73.75 \%$ global publication share during 2007-16. Their five-yearly output accounted for $71.88 \%$ global publication share during $2007-11$ which increased to $75.36 \%$ during succeeding 5 -year period 2012-16. Each of top 10 countries accounted for $3.67 \%$ to $25.88 \%$ global publication share during 2007-16, with USA accounting for the highest publication share (25.88\%), followed by Italy (8.15\%),China (7.70\%), U.K., Germany and Japan (from $5.41 \%$ to $5.54 \%$ ), South Korea (4.29\%), France, Turkey and India (from $3.67 \%$ to $3.82 \%$ ) during 2007-16. The global publication

\begin{tabular}{|c|c|c|c|c|c|c|c|}
\hline \multirow[t]{2}{*}{ Year } & \multirow{2}{*}{$\begin{array}{c}\text { World } \\
\text { TP }\end{array}$} & \multicolumn{6}{|c|}{ India } \\
\hline & & TP & $\mathrm{TC}$ & CPP & \%ТP & ICP & $\% I C P$ \\
\hline 2007 & 5489 & 140 & 1216 & 8.69 & 2.55 & 8 & 5.71 \\
\hline 2008 & 5615 & 138 & 1886 & 13.67 & 2.46 & 9 & 6.52 \\
\hline 2009 & 5869 & 175 & 1473 & 8.42 & 2.98 & 15 & 8.57 \\
\hline 2010 & 6324 & 221 & 1989 & 9.00 & 3.49 & 21 & 9.50 \\
\hline 2011 & 6713 & 278 & 1940 & 6.98 & 4.14 & 25 & 8.99 \\
\hline 2012 & 6971 & 264 & 1225 & 4.64 & 3.79 & 32 & 12.12 \\
\hline 2013 & 7355 & 261 & 849 & 3.25 & 3.55 & 33 & 12.64 \\
\hline 2014 & 7680 & 358 & 737 & 2.06 & 4.66 & 37 & 10.34 \\
\hline 2015 & 7416 & 325 & 423 & 1.30 & 4.38 & 25 & 7.69 \\
\hline 2016 & 5560 & 323 & 51 & 0.16 & 5.81 & 14 & 4.33 \\
\hline $2007-11$ & 30010 & 952 & 8504 & 8.93 & 3.17 & 78 & 8.19 \\
\hline $2012-16$ & 34982 & 1531 & 3285 & 2.15 & 4.38 & 141 & 9.21 \\
\hline $2007-16$ & 64992 & 2483 & 11789 & 4.75 & 3.82 & 219 & 8.82 \\
\hline
\end{tabular}

TP=Total Papers; TC=Total Citations; $\mathrm{CPP}=$ Citations Per Paper 
share in five years increased by $5.67 \%$ by China, followed by $2.38 \%$ by South Korea, $0.92 \%$ by India and $0.40 \%$ by Turkey as against decrease by $2.28 \%$ by USA, $1.12 \%$ by U.K., $1.0 \%$ by Germany, $0.79 \%$ by France, $0.62 \%$ by Japan and $0.06 \%$ by Italy from $2007-11$ to $2012-16$.

\section{Subject-Wise Distribution of Research Output}

The Indian thyroid research output published during 2007-16 is distributed across five sub-fields (as identified in Scopus database classification), with medicine accounting for the highest publications share (77.57\%), followed by biochemistry, genetics \& molecular biology (23.60\%), pharmacology, toxicology \& pharmaceutics (8.46\%), agricultural \& biological sciences (3.34\%) and neurosciences (2.54\%) during 2007-16. The activity index, which computes change in research activity in a discipline over time 2007-11 to 2012-16 (world average activity index of a given subject is taken as 100), witnessed increase in biochemistry, genetics \& molecular biology (from 89.46 to $106.55 \%$ ) and pharmacology, toxicology \& pharmaceutics (from 88.18 to 107.35), as against decline of research activity in medicine (from 101.70 to 98.94 ), agricultural \& biological sciences (from 135.12 to 78.16) and neurosciences (from 124.20 to 84.95) from 2007-11 to 2012-16. Agricultural \& biological sciences registered the highest citation impact per paper of 7.22 , followed by neurosciences (6.24), biochemistry, genetics \& molecular biology (6.12), pharmacology, toxicology \& pharmaceutics (5.85) and medicine (4.65) during 2006-15 (Table 4).

\section{Sub-Field Wise Classification of Thyroid Research}

In terms of sub-specialties, clinical thyroidology received the largest share $(65.85 \%)$ of papers, followed by thyroid Interface with non-endocrine systems (23.28\%), investigational thyroidology (11.50\%) and thryroid interface with endocrine systems (5.80\%) during 2007-16. Within clinical thyroidology, the major share $(32.46 \%)$ was contributed by oncology thyroidology, followed by medical thyroidology $(19.73 \%)$, surgical thyroidology (10.78\%), etc [Table 5].

\section{Profile of Top 15 Most Productive Global Organizations}

The productivity of 15 most productive global organizations in Indian thyroid research varied from 24 to 159 publications and together they

\begin{tabular}{|c|c|c|c|c|c|c|}
\hline \multirow{2}{*}{$\begin{array}{c}\text { Collaborative } \\
\text { Country }\end{array}$} & \multicolumn{3}{|c|}{ Number of Papers } & \multicolumn{3}{|c|}{ Share of Papers } \\
\hline & 2007-11 & 2012-16 & 2007-16 & 2007-11 & 2012-16 & 2007-16 \\
\hline USA & 31 & 77 & 108 & 39.74 & 54.61 & 49.32 \\
\hline Italy & 7 & 16 & 23 & 8.97 & 11.35 & 10.50 \\
\hline U.K. & 12 & 11 & 23 & 15.38 & 7.80 & 10.50 \\
\hline Germany & 5 & 12 & 17 & 6.41 & 8.51 & 7.76 \\
\hline France & 8 & 7 & 15 & 10.26 & 4.96 & 6.85 \\
\hline Japan & 6 & 9 & 15 & 7.69 & 6.38 & 6.85 \\
\hline Netherlands & 5 & 10 & 15 & 6.41 & 7.09 & 6.85 \\
\hline Australia & 8 & 6 & 14 & 10.26 & 4.26 & 6.39 \\
\hline China & 6 & 7 & 13 & 7.69 & 4.96 & 5.94 \\
\hline Saudi Arabia & 2 & 9 & 11 & 2.56 & 6.38 & 5.02 \\
\hline Indian Total & 78 & 141 & 219 & 100.00 & 100.00 & 100.00 \\
\hline
\end{tabular}

\begin{tabular}{ccccccc}
$\begin{array}{c}\text { Table 3: Global Publication Share of Top } \mathbf{1 0} \text { Most Productive Countries in Throid Research during 2007-16 } \\
\text { Name of the Country }\end{array}$ & \multicolumn{7}{c}{ Shmare of Papers } \\
& $2007-11$ & $2012-16$ & $2007-16$ & $2007-11$ & $2012-16$ & $2007-16$ \\
USA & 8137 & 8686 & 16823 & 27.11 & 24.83 & 25.88 \\
Italy & 2457 & 2843 & 5300 & 8.19 & 8.13 & 8.15 \\
China & 1393 & 3609 & 5002 & 4.64 & 10.32 & 7.70 \\
U.K. & 1842 & 1756 & 3598 & 6.14 & 5.02 & 5.54 \\
Germany & 1810 & 1759 & 3569 & 6.03 & 5.03 & 5.49 \\
Japan & 1725 & 1793 & 3518 & 5.75 & 5.13 & 5.41 \\
South Korea & 903 & 1884 & 2787 & 3.01 & 5.39 & 4.29 \\
France & 1274 & 1207 & 2481 & 4.25 & 3.45 & 3.82 \\
Turkey & 1077 & 1394 & 2471 & 3.59 & 3.98 & 3.80 \\
India & 952 & 1431 & 2383 & 3.17 & 4.09 & 3.67 \\
Total & 21570 & 26362 & 47932 & 71.88 & 75.36 & 73.75 \\
World & 30010 & 34982 & 64992 & 100.00 & 100.00 & 100.00
\end{tabular}




\begin{tabular}{|c|c|c|c|c|c|c|c|c|}
\hline \multirow[t]{2}{*}{ Subject* } & \multicolumn{3}{|c|}{ Number of Papers (TP) } & \multicolumn{2}{|c|}{ Activity Index } & \multirow{2}{*}{$\begin{array}{c}\text { TC } \\
\text { 2007-16 }\end{array}$} & \multirow{2}{*}{$\begin{array}{c}\text { CPP } \\
2007-16\end{array}$} & \multirow{2}{*}{$\begin{array}{c}\% \text { TP } \\
2007-16\end{array}$} \\
\hline & 2007-11 & 2012-16 & 2007-16 & 2007-11 & 2012-16 & & & \\
\hline Medicine & 751 & 1175 & 1926 & 101.70 & 98.94 & 8955 & 4.65 & 77.57 \\
\hline $\begin{array}{l}\text { Biochemistry, Genetics \& } \\
\text { Molecular Biology }\end{array}$ & 201 & 385 & 586 & 89.46 & 106.55 & 3584 & 6.12 & 23.60 \\
\hline $\begin{array}{c}\text { Pharmacology, Toxicology \& } \\
\text { Pharmaceutics }\end{array}$ & 71 & 139 & 210 & 88.18 & 107.35 & 1229 & 5.85 & 8.46 \\
\hline Agricultural \& Biological Sciences & 43 & 40 & 83 & 135.12 & 78.16 & 599 & 7.22 & 3.34 \\
\hline Neurosciences & 30 & 33 & 63 & 124.20 & 84.95 & 393 & 6.24 & 2.54 \\
\hline Total Indian Output & 952 & 1531 & 2483 & 100.00 & 100.00 & & & \\
\hline
\end{tabular}

\begin{tabular}{|c|c|c|c|c|c|c|c|c|}
\hline \multirow[t]{2}{*}{ Sub-Speciality } & \multicolumn{3}{|c|}{ Number of Papers } & \multicolumn{3}{|c|}{ Share of Papers } & \multirow[t]{2}{*}{ TC } & \multirow[t]{2}{*}{ CPP } \\
\hline & 2007-11 & 2012-16 & 2007-16 & 2007-11 & 2012-16 & 2007-16 & & \\
\hline Clinical Thyroidology & 643 & 992 & 1635 & 67.54 & 64.79 & 65.85 & 8568 & 5.24 \\
\hline Medical Thyroidology & 106 & 302 & 408 & 11.13 & 19.73 & 16.43 & 1554 & 3.81 \\
\hline Pediatric Thyroidology & 22 & 49 & 71 & 2.31 & 3.20 & 2.86 & 316 & 4.45 \\
\hline Obstetric Thyroidology & 6 & 19 & 25 & 0.63 & 1.24 & 1.01 & 161 & 6.44 \\
\hline Surgical Thyroidology & 101 & 165 & 266 & 10.61 & 10.78 & 10.71 & 863 & 3.24 \\
\hline Autoimmunity Thyroidology & 15 & 27 & 42 & 1.58 & 1.76 & 1.69 & 358 & 8.52 \\
\hline Oncology Thyroidology & 331 & 497 & 828 & 34.77 & 32.46 & 33.35 & 4242 & 5.12 \\
\hline Investigational Thyroidology & 75 & 176 & 251 & 7.88 & 11.50 & 10.11 & 994 & 3.96 \\
\hline Biochemistry/Essay & 24 & 47 & 71 & 2.52 & 3.07 & 2.86 & 284 & 4.00 \\
\hline Pathology & 48 & 119 & 167 & 5.04 & 7.77 & 6.73 & 673 & 4.03 \\
\hline Nuclear Medicine & 2 & 5 & 7 & 0.21 & 0.33 & 0.28 & 33 & 4.71 \\
\hline Radiology & 1 & 5 & 6 & 0.11 & 0.33 & 0.24 & 4 & 0.67 \\
\hline Thryroid Interface with Endocrine Systems & 56 & 88 & 144 & 5.88 & 5.75 & 5.80 & 1297 & 9.01 \\
\hline Vitamin D and Musculoskeletal & 11 & 21 & 32 & 1.16 & 1.37 & 1.29 & 201 & 6.28 \\
\hline $\begin{array}{c}\text { Obesity/Metabolic Syndrome/Cardiovascular } \\
\text { Systems }\end{array}$ & 7 & 15 & 22 & 0.74 & 0.98 & 0.89 & 321 & 14.59 \\
\hline Pancreas & 21 & 33 & 54 & 2.21 & 2.16 & 2.17 & 619 & 11.46 \\
\hline Pituitary & 17 & 19 & 36 & 1.79 & 1.24 & 1.45 & 156 & 4.33 \\
\hline Thyroid Interface with Non-Endocrine Systems & 260 & 318 & 578 & 27.31 & 20.77 & 23.28 & 3746 & 6.48 \\
\hline Nervous System & 17 & 21 & 38 & 1.79 & 1.37 & 1.53 & 533 & 14.03 \\
\hline Eye & 14 & 23 & 37 & 1.47 & 1.50 & 1.49 & 164 & 4.43 \\
\hline Skin & 47 & 67 & 114 & 4.94 & 4.38 & 4.59 & 696 & 6.11 \\
\hline Hematology & 2 & 4 & 6 & 0.21 & 0.26 & 0.24 & 1 & 0.17 \\
\hline Liver & 94 & 103 & 197 & 9.87 & 6.73 & 7.93 & 1873 & 9.51 \\
\hline Dental & 1 & 3 & 4 & 0.11 & 0.20 & 0.16 & 229 & 57.25 \\
\hline Kidney & 85 & 97 & 182 & 8.93 & 6.34 & 7.33 & 250 & 1.37 \\
\hline Total of India & 952 & 1531 & 2483 & 100.00 & 100.00 & 100.00 & & \\
\hline
\end{tabular}


contributed 31.70\% (787) publication share and 34.72\% (4093) citation share to its cumulative publications output during 2007-16. The scientometric profile of these 20 organizations is presented in Table 6. Four of these organizations registered publications output greater than the group average of 52.47: AIIMS - New Delhi (159 papers), PGIMERChandigarh and TMH-Mumbai (94 papers each) and SGPGIMS-Lucknow (92 papers) during 2007-16. Four organizations registered impact above the group average of 5.20 citations per publication during 2007-16: INMAS-Delhi (11.26), AIIMS-New Delhi (7.70), BARC-Mumbai (6.71) and SGPGIMS-Lucknow (5.82) during 2007-16. Six organizations registered h-index above the group average of 7.93: AIIMS-New Delhi (16), INMAS-Delhi and SGPGIMS-Lucknow (13 each), TMH-Mumbai (11), PGIMER-Chandigarh (8) and JIPMER- Pondicherry (8) during 2007-16. Seven organizations contributed international collaborative publications share above the group average of 5.59\%: University of Calcutta, Kolkata and MAMC-Delhi (14.29\% each), KMC-Manipal (11.54\%), TMHMumbai (8.51\%), SIKIMS-Srinagar (7.69\%), BARC-Mumbai $(6.45 \%)$ and AIIMS-New Delhi (5.66\%) during 2007-16. Four organizations registered the relative citation index above the group average (1.09) of all organizations: INMAS-Delhi (2.37), AIIMS-New Delhi (1.62), BARCMumbai (1.41) and SGPGIMS- Lucknow (1.22) during 2007-16.

\section{Profile of Top 15 Most Productive Authors}

The research productivity of top 15 most productive authors in Indian thyroid research varied from 12 to 34 publications. Together they contributed $11.24 \%$ (279) Indian publication share and $17.58 \%$ (2072) cita- tion share during 2007-16. The scientometric profile of these 15 authors is presented in Table 7. Four authors registered publications output above the group average of 18.60: S. Basu (34 papers), A. Agarwal (27 papers), C. Bal (31 papers) and R.K. Marwaha (19 papers) during 2007-16. Six authors registered impact above the group average of 7.43 citations per publication: A.Kar (24.41), R.K.Marwaha (16.26), G. Mugesh (12.29), N. Tandon (11.94), G.Agarwal (11.38) ANDA.Bhansali (8.58) during 2007-16. Six authors registered h-index above the group average of 6.27 of all authors: A.Kar (11), R.K.Marwaha (10), N.Tandon and C.Bal (8 each), G.Mugesh and G.Agarwal (7 each) during 2007-16. Four authors contributed international collaborative publications share above the group average of $6.09 \%$ of all authors: S.Basu (20.59\%), C.Bal (12.90\%), G.Mugesh (11.76\%) and B.R.Mittal (6.67\%) during 2007-16. Six authors registered the relative citation index above the group average (1.56) of all authors: A.Kar (5.14), R.K.Marwaha (3.42), G.Mugesh (2.59), N.Tandon and G.Agarwal (2.39 each) and A.Bhansali (1.81) during 2007-16.

\section{Medium of Communication}

Of the total Indian output in thyroid research, 98.35\% (2343) appeared in journals. The top 15 most productive journals accounted for 22 to 95 papers each in thyroid research and together accounted for $23.23 \%$ share (544 papers) of total journal publication output during 2007-16. The publication share of these top 15 most productive journals increased from $19.62 \%$ to $25.66 \%$ between $2007-11$ and $2012-16$. The top most productive journal (with 95 papers) was Journal of Clinical \& Diagnostic Research, followed by Indian Journal of Nuclear Medicine (51 papers),

\begin{tabular}{|c|c|c|c|c|c|c|c|}
\hline Name of the Organization & TP & TC & CPP & $\mathrm{HI}$ & ICP & \%ICP & $\mathrm{RCl}$ \\
\hline $\begin{array}{l}\text { All India Institute of Medical Sciences (AIIMS), New } \\
\qquad \text { Delhi }\end{array}$ & 159 & 1225 & 7.70 & 16 & 9 & 5.66 & 1.62 \\
\hline $\begin{array}{l}\text { Postgraduate Institute of Medical Education \& } \\
\text { Research (PGIMER), Chandigarh }\end{array}$ & 94 & 367 & 3.90 & 10 & 2 & 2.13 & 0.82 \\
\hline Tata Memorial Hospital (TMH), Mumbai & 94 & 369 & 3.93 & 11 & 8 & 8.51 & 0.83 \\
\hline $\begin{array}{l}\text { Sanjay Gandhi Postgraduate Institute of Medical } \\
\text { Sciences (SGPGIMS), Lucknow }\end{array}$ & 92 & 535 & 5.82 & 13 & 5 & 5.43 & 1.22 \\
\hline Christian Medical College (CMC), Vellore & 45 & 84 & 1.87 & 5 & 2 & 4.44 & 0.39 \\
\hline $\begin{array}{l}\text { Institute of Nuclear Medicine \& Allied Sciences } \\
\text { (INMAS), Delhi }\end{array}$ & 43 & 484 & 11.26 & 13 & 1 & 2.33 & 2.37 \\
\hline $\begin{array}{l}\text { Jawaharlal Institute of Postgraduate Medical } \\
\text { Education \& Research (JIPMER), Pondicherry }\end{array}$ & 39 & 192 & 4.92 & 8 & 0 & 0.00 & 1.04 \\
\hline $\begin{array}{l}\text { PBDS Postgraduate Institute of Medical Sciences } \\
\text { (PBDSPGIMS), Rohtak }\end{array}$ & 33 & 128 & 3.88 & 6 & 1 & 3.03 & 0.82 \\
\hline Bhabha Atomic Research Center (BARC), Mumbai & 31 & 208 & 6.71 & 7 & 2 & 6.45 & 1.41 \\
\hline Maulana Azad Medical College (MAMC), Delhi & 28 & 53 & 1.89 & 4 & 4 & 14.29 & 0.40 \\
\hline University of Calcuta, Kolkata & 28 & 94 & 3.36 & 6 & 4 & 14.29 & 0.71 \\
\hline $\begin{array}{l}\text { Sher-I-Kashmir Institute of Medical Sciences } \\
\text { (SIKIMS), Srinagar }\end{array}$ & 26 & 129 & 4.96 & 6 & 2 & 7.69 & 1.04 \\
\hline Kasturba Medical College (KMC), Manipal & 26 & 38 & 1.46 & 3 & 3 & 11.54 & 0.31 \\
\hline Lady Harding Medical College (LHMC), New Delhi & 25 & 114 & 4.56 & 6 & 0 & 0.00 & 0.96 \\
\hline Medical College \& Hospital (MCH), Kolkata & 24 & 73 & 3.04 & 5 & 1 & 4.17 & 0.64 \\
\hline Total of 15 organizations & 787 & 4093 & 5.20 & 7.93 & 44 & 5.59 & 1.09 \\
\hline Total of India & 2483 & 11789 & 4.75 & & & & \\
\hline Share of top 15 organizations in India total output & 31.70 & 34.72 & & & & & \\
\hline
\end{tabular}

TP=Total Papers; TC=Total Citations; $\mathrm{CPP}=$ Citations Per Paper; HI=h-index; ICP=International Collaborative Papers; RCI=Relative Citation Index 


$\begin{array}{cccccccccc}\text { Table 7: Scientometric Profile of Top 15 Most Productive Authors in Thyroid Research in India during 2007-16 } \\ \begin{array}{c}\text { Name of the } \\ \text { Author }\end{array} & \text { Affiliation of the Author } & \text { TP } & \text { TC } & \text { CPP } & \text { HI } & \text { ICP } & \text { \%ICP } & \text { RCI } \\ \text { S.Basu } & \text { TMH-Mumbai } & 34 & 96 & 2.82 & 6 & 7 & 20.59 & 0.59 \\ \text { A.Agarwal } & \text { SGPIMS-Lucknow } & 27 & 76 & 2.81 & 6 & 1 & 3.70 & 0.59 \\ \text { C.Bal } & \text { AIIMS-New Delhi } & 31 & 169 & 5.45 & 8 & 4 & 12.90 & 1.15 \\ \text { R.K.Marwaha } & \text { INMAS-Delhi } & 19 & 309 & 16.26 & 10 & 0 & 0.00 & 3.42 \\ \text { A.Mishra } & \text { SGPIMS-Lucknow } & 18 & 71 & 3.94 & 6 & 1 & 5.56 & 0.83 \\ \text { N.Tandon } & \text { AIIMS-New Delhi } & 18 & 215 & 11.94 & 8 & 0 & 0.00 & 2.51 \\ \text { A.Kar } & \text { Devi Ahila Uniersity, Indore } & 17 & 415 & 24.41 & 11 & 1 & 5.88 & 5.14 \\ \text { G.Mugesh } & \text { IISc-Bangalore } & 17 & 209 & 12.29 & 7 & 2 & 11.76 & 2.59 \\ \text { G.Agarwal } & \text { SGPIMS-Lucknow } & 16 & 182 & 11.38 & 7 & 0 & 0.00 & 2.39 \\ \text { B.R.Mittal } & \text { PGIMER-Chandigarh } & 15 & 44 & 2.93 & 4 & 1 & 6.67 & 0.62 \\ \text { P.V.Pradeep } & \text { SGPIMS-Lucknow } & 15 & 56 & 3.73 & 4 & 0 & 0.00 & 0.79 \\ \text { A.Bhattacharya } & \text { PGIMER-Chandigarh } & 14 & 27 & 1.93 & 4 & 0 & 0.00 & 0.41 \\ \text { S.K.Mishra } & \text { SGPIMS-Lucknow } & 14 & 84 & 6.00 & 6 & 0 & 0.00 & 1.26 \\ \text { A.Abhyankar } & \text { TMH-Mumbai } & 12 & 16 & 1.33 & 2 & 0 & 0.00 & 0.28 \\ \text { A.Bhansali } & \text { PGIMER-Chandigarh } & 12 & 103 & 8.58 & 5 & 0 & 0.00 & 1.81 \\ & \text { Total of 15 authors } & 279 & 2072 & 7.43 & 6.27 & 17 & 6.09 & 1.56\end{array}$

TP=Total Papers; TC=Total Citations; CPP=Citations Per Paper; HI=h-index; ICP=International Collaborative Papers; RCI=Relative Citation Index

\begin{tabular}{|c|c|c|c|}
\hline \multirow[t]{2}{*}{ Name of the Journal } & \multicolumn{3}{|c|}{ Number of Papers } \\
\hline & 2007-11 & 2012-16 & 2007-16 \\
\hline Journal of Clinical \& Diagnostic Research & 12 & 83 & 95 \\
\hline Indian Journal of Nuclear Medicine & 17 & 34 & 51 \\
\hline BMJ Case Reports & 2 & 42 & 44 \\
\hline Indian Journal of Pathology \& Microbiology & 25 & 15 & 40 \\
\hline Indian Journal of Endocrinology \& Metabolism & 0 & 36 & 36 \\
\hline Journal of Association of Physicians of India & 22 & 11 & 33 \\
\hline Journal of Cytology & 13 & 20 & 33 \\
\hline Journal of Cancer Research \& Therapy & 10 & 22 & 32 \\
\hline International Journal of Pharma \& Bio Sciences & 3 & 27 & 30 \\
\hline Clinical Nuclear Medicine & 15 & 13 & 28 \\
\hline Indian Journal of Cancer & 13 & 14 & 27 \\
\hline Indian Journal of Surgical Oncology & 17 & 10 & 27 \\
\hline World Journal of Surgery & 13 & 11 & 24 \\
\hline Indian Journal of Dermatology & 12 & 10 & 22 \\
\hline Indian Journal of Pediatrics & 11 & 11 & 22 \\
\hline Total of 15 journals & 185 & 359 & 544 \\
\hline Total India journal output & 943 & 1399 & 2342 \\
\hline Share of top 15 journals in Indian journal output & 19.62 & 25.66 & 23.23 \\
\hline
\end{tabular}




\begin{tabular}{|c|c|c|c|c|}
\hline Keyword & Frequency & S.No & Keyword & Frequency \\
\hline Thyroxine & 477 & 23 & Thyroid Papillary Carcinoma & 122 \\
\hline Thyrotropin & 436 & 24 & Immunochemistry & 115 \\
\hline Hypothyrodism & 429 & 25 & Thyroid Tumor & 113 \\
\hline Thyroid Hormone & 356 & 26 & Blood & 109 \\
\hline Thyroid Gland & 339 & 27 & Metabolism & 108 \\
\hline Liothyronine & 336 & 28 & Levothyroxine & 105 \\
\hline Thyroidectomy & 312 & 29 & Aspiration Cytology & 87 \\
\hline Thyroid Function Test & 301 & 30 & Pregnancy & 87 \\
\hline Thyroid Disease & 297 & 31 & Protein Expression & 87 \\
\hline Thyroid Neoplasm & 264 & 32 & Thorax Radiography & 86 \\
\hline Echography & 217 & 33 & Thyroglobulin & 85 \\
\hline Throtopin Blood Level & 210 & 34 & Thyroid Medullary Carcinoma & 82 \\
\hline Thyroid & 190 & 35 & Lymph Mode Metastasis & 80 \\
\hline Throxine Blood & 176 & 36 & Breast Cancer & 79 \\
\hline Thyroid Cancer & 173 & 37 & Thyroid Scientscanning & 77 \\
\hline Hyperthyroidism & 172 & 38 & Hypertension & 73 \\
\hline Goiter & 168 & 39 & Thyrotoxicosis & 72 \\
\hline Thyroid Carcinoma & 161 & 40 & Thyroid Follicular Carcinoma & 71 \\
\hline Pathology & 156 & 41 & Diabetes Mellitus & 69 \\
\hline NML Resonance Imaging & 140 & 42 & Pathophysiology & 69 \\
\hline Thyroid Nodule & 135 & 43 & Hydrocortisone & 67 \\
\hline Iodine 131 & 126 & & & \\
\hline
\end{tabular}

BMJ Case Reports (44 papers), Indian Journal of Pathology \& Microbiology (40 papers), etc. during 2007-16 (Table 8).

\section{Significant Keywords}

Around 43 significant keywords have been identified from the literature, which point to possible trends in mobile cloud computing research. These keywords are listed in Table 9 in the decreasing order of the frequency of occurrence during 2007-16.

\section{Highly Cited Papers}

A total of 13 highly cited papers were identified which received citations from 83 to 422 ( 5 papers in citation range 83-97, 5 papers in citation range 108-139 and 3 papers in citation range 218-422) during 200716. These 13 papers together received 2023 citations, which averaged to 155.61 citations per paper. Of the 13 highly cited papers, 2 resulted from the participation of single organization (non-collaborative) and 11 involved the participation of two or more organizations ( 2 national collaborative and 9 international collaborative). Among international collaborative papers, the largest participation was from USA (6 papers), followed by France, Argentina and Netherlands (3 papers each), U.K., Belgium, Mexico, Switzerland, Germany, Japan, China and Australia (2 papers each) and 1 paper each by 22 countries. There were 17 Indian organizations involved in these high cited papers. Of the 13 highly cited papers, 8 were published as articles, 3 as review papers and 2 as conference paper. These 13 highly cited papers were published in 11 journals, of which 2 papers were published in American Journal of Transplantation and 1 paper each in 10 other journals, namely Archives of Medical Science, BJOG: An International Journal of Obstetrics \& Gynaecology, Fitoterpia, International Journal of Epidemiology, Journal of Allergy \&
Clinical Immunology, Journal of Gastroentrology \& Heptatology, The Lancet, Pharmacognosy Review, Silence, World Journal of Surgery and World Psychiatry.

\section{CONCLUSION}

Using the Scopus database, this study provides a quantitative and qualitative description of the development of the research involving thyroid research over a period of 10 years. From 2007 to 2016 the scientific literature related to thyroid research registered a high growth of $10.76 \%$ per annum. However, qualitative performance of thyroid research measured in terms of citation impact is not as stunning. Compared to quantitative performance it averaged to mere 4.75 citations per paper in 10 years period. India's share in global output on thyroid research was $8.82 \%$, which increased from $8.19 \%$ to $9.21 \%$ from $2007-11$ to $2012-16$. USA is the top most productive country in the world in thyroid research. The other Asian countries joining the top 10 most productive countries in 200716: China (ranked $\left.3^{\text {rd }}\right)$, Japan $\left(6^{\text {th }}\right)$, South Korea $\left(7^{\text {th }}\right)$ and India $\left(10^{\text {th }}\right)$. From the western world and North America, the USA ranked $1^{\text {st }}$, Italy $\left(2^{\text {nd }}\right.$ rank $)$, U.K. ( $4^{\text {th }}$ rank $)$, Germany $\left(5^{\text {th }}\right.$ rank $)$, France $\left(8^{\text {th }}\right.$ rank $)$ and Turkey ( $9^{\text {th }}$ rank).

Medicine among subjects accounted for highest publication share of $77.57 \%$, followed by biochemistry, genetics \& molecular biology (23.60\%), pharmacology, toxicology \& pharmaceutics (8.46\%), agricultural \& biological sciences (3.34\%) and neurosciences (2.54\%) during 2007-16. The research activity witnessed increase in by biochemistry, genetics \& molecular biology and pharmacology, toxicology \& pharmaceutics, as against decline of research activity in medicine, agricultural \& biological sciences and neurosciences from 2007-11 to 2012-16. Agri- 
cultural \& biological sciences registered the highest citation impact per paper of 7.22, followed by neurosciences (6.24), biochemistry, genetics \& molecular biology (6.12), pharmacology, toxicology \& pharmaceutics (5.85) and medicine (4.65) during 2006-15 .

In terms of sub-specialties, clinical thyroidology received the largest share $(65.85 \%)$ of papers, followed by thyroid Interface with non-endocrine systems (23.28\%), investigational thyroidology (11.50\%) and thryroid interface with endocrine systems (5.80\%) during 2007-16.

The top 15 most productive research organizations and the authors collectively contributed $31.70 \%$ and $11.24 \%$ respectively as their global publication share and $34.72 \%$ and $17.58 \%$ respectively as their global citation share during 2007-16.

The journals as a medium ranked $1^{\text {st }}$, accounting for $98.35 \%$ share of total Indian output on thyroid research during 2007-16. The 15 most productive journals involved in Indian thyroid research constituted 23.23\% share of total journal publication output during 2007-16, increasing from $19.62 \%$ to $25.66 \%$ between $2007-11$ and 2012-16. Journal of Clinical \& Diagnostic Research accounted for largest number of papers (95), followed by Indian Journal of Nuclear Medicine (51 papers), BMJ Case Reports (44 papers), Indian Journal of Pathology \& Microbiology (40 papers), etc. during 2007-16.

Of the total Indian output on thyroid research, only 13 publications registered high citations, in the range of 83 to 422 citations per paper, and collectively these 13 highly cited papers received a total of 2023 citations, averaging to 155.61 citations per paper. Among international collaborative papers, the largest participation was from USA (6 papers), followed by France, Argentina and Netherlands (3 papers each), U.K., Belgium, Mexico, Switzerland, Germany, Japan, China and Australia (2 papers each) and 1 paper each by 22 countries. There were 17 Indian organiza- tions involved in these high cited papers. Of the 13 highly cited papers, 8 were published as articles, 3 as review papers and 2 as conference paper. These 13 highly cited papers were published in 11 journals, of which 2 papers were published in American Journal of Transplantation and 1 paper each in 10 other journals

Concludes that there is a high burden of thyroid diseases in India, which is under-addressed both within government programs, population level and the medical fraternity level too. Thyroid diseases are different from other diseases in terms of their diagnosis and medical treatment. Early diagnosis and treatment is the cornerstone of management of thyroid disorders, hence there is an urgent need to build capacity in this area as thyroid has become a serious health threat with exploding population. India needs to build capacity amongst primary care physicians to address management, counseling, referral and prevention of thyroid disorders. It will go a step further to tackle the rising prevalence thyroid disorders aimed to strengthen the capacity of doctors to deal with the enormous challenges it poses, with the ultimate aim of improving the health outcomes of people.

\section{REFERENCES}

1. Stoppler, Melissa Conrad. What are thyroid disorders? 1996-2017 MedicineNet, Inc. http://www.medicinenet.com/thyroid_disorders/article.htm

2. Business Wire India. First of its Kind Certificate Course in Management of Thyroid Disorders (CCMTD) Launched Nationally. June 21, 2015. http://www.financialexpress.com/industry/first-of-its-kind-certificate-course-in-managementof-thyroid-disorders-ccmtd-launched-nationally/87626/.

3. Unnikrishnan AG, Menon UV. Thyroid disorders in India: An epidemiological perspective. Indian J Endocr Metab [serial online] 2011;15, Suppl S2:78-81. Available from: http://www.ijem.in/text.asp?2011/15/6/78/83329

4. Bhutani, Garima, Verma, Prem, Kalra, Sanjay. Bibliometrics analyses of Thyroid Research and Practice. Thyroid Research \& Practice. 2014;11(1):17-21

Cite this article : Gupta BM, Mueen Ahmed KK, Gupta R. Thyroid Research in India: A Scientometric Assessment of Publications Output during 2007-16. Int J Med Public Health. 2017;7(3):134-41. 